\title{
The Moderating Role of Social Media Platforms on Self-Esteem and Life Satisfaction: A Case Study of YouTube and Instagram
}

\author{
Kathy Tian \\ The University of Illinois at Urbana-Champaign \\ Hong Zhu \\ Loyola University Maryland \\ Tian Guang \\ (Corresponding Author) \\ Huaihua University
}

Social media use has become increasingly prolific in modern society, and, as a by-product, so too have the negative implications of excessive social media use. Although there exists a robust body of research on social media use and its subsequent association with social media addiction, life satisfaction and selfesteem, few studies have examined how the social media medium (i.e., the social media platform itself) may influence users distinctively. This study explores how medium differences on the social media platforms, Instagram and YouTube, may result in different media effects for social media users. It is expected that engaging with YouTube may increase users' self-esteem and life satisfaction or that preexposure and post-exposure measures will remain constant. However, it is predicted that engaging with Instagram will decrease self-esteem and measures of life-satisfaction post-exposure. Findings of this study can be widely applied in modern business practice.

Keywords: Social Media, Media Addiction, Self-esteem, Life Satisfaction

\section{INTRODUCTION}

The use of social media has witnessed exponential growth in the period of less than one decade; as of January 2017, social media use is estimated to engage roughly 2.80 billion people, or over one third of the world's population (WeAreSocial, 2017). In recent years, social media engagement has developed into an increasingly popular form of entertainment across the globe (Kuss \& Griffiths, 2011), with users seeking various gratifications through social media participation, from communication and connectivity to social affirmation and information sharing (see Quan-Hasse \& Young, 2010; Toma \& Hancock, 2013; Quinn, 2016). Although activities such as sharing personal information, maintaining social connections, and distracting oneself through social media are regarded as mundane phenomena, concerns have been raised 
about the potentially addictive attributes of social media as well as its subsequent impact on the psychosocial well-being of consumers (Andreassen, Pallesen, Griffiths, 2017).

\section{LITERATURE REVIEW}

\section{Social Media and Addiction}

Social media platforms, as industries profiting from prolific user engagement and information distribution, are mediums that inherently encourage overuse. Restricted self-direction, in combination with youths' innate desire for acceptance and companionship, has fostered some adolescents to rely on social media for validation, resulting in both significant health and psychological issues amongst susceptible users (Balakrishnan \& Griffiths, 2017). Previous research suggests that extensive use of social networking tools affects various aspects of users' daily life in terms of psychosomatic well-being, overall health, and family and work life.

Addictive social media behavior can be distinguished from non-addictive behavior as the former is defined by excessive and compulsory use of such mediums while the latter involves more personal directive in social media use (Andreassen et al., 2017). General addiction models have been applied to explain the obsessive habitual use of social media by afflicted individuals (Griffiths, 2005). Specifically, the state of networking addiction is defined to be characterized by uncontrollable desires to access social media, inordinate concern about social media, and the tendency to devote excessive time toward social media to the extent that it results in the deterioration of other integral dimensions of personal and public life (Andreassen \& Pallesen, 2014). Addictive media use further manifests as a preoccupation with social media, gradually developing cravings for the pleasure derived from social media use and exhibitions of withdrawal symptoms when prohibited from such interactive media platforms (Andreassen et al., 2017).

Considering the significant ramifications social media use may have on an individual's personal welfare, it is important to exam the various factors that influence addiction as well as the role these factors play in moderating addictive tendencies. A robust body of research suggests that individual differences predict the propensity for social networking addiction. Dispositional differences in attachment style, for instance, predict Facebook use; highly attached individuals demonstrate more social ties and boast larger social networks than unattached counterparts (Jenkins-Guarnieri, Wright, \& Hudiburgh, 2012) and anxiously attached individuals tend to be more concerned about how social others perceive them on Facebook, in addition to using the platform more habitually (Lin 2015, 2016). Various other idiosyncratic factors have also been found to predict addiction to social networking sites, from gender (Andreassen et al., 2017) to fear of missing out, or FOMO (Blackwell, Leaman, Tramposch, Osborne, \& Liss, 2017).

In terms of demographics, studies generally report higher levels of social media addiction in younger users compared to older users (Kuss et al., 2014), singles compared to those in a committed relationship, and higher scores for addiction in women over men (Griffiths et al., 2014). Additionally, in examining data collected from a national survey of 23,500 Norwegian participants on addictive social media use, Andreassen et al. (2017) identified several individual differences associated with addictive behaviors. Their analyses suggest that compulsive actions are related to higher levels of narcissism as well as lower levels of self-esteem; they posit that social media may be a more gratifying medium for narcissistic individuals and individuals with low self-esteem may find social media a safer place for self-expression. Interestingly, Blackwell et al. (2017) also suggest that factors such as self-esteem and neuroticism are predictive of social media use, but only fear of missing out (FOMO) was significantly predictive of addiction.

\section{Self-esteem}

Further exacerbating the concerns over social media addiction are the symptoms associated with addictive tendencies, which negatively affect aspects of mental health including subjective well-being and self-esteem (Pantic, 2014; Hawi \& Samaha, 2017). Studies exploring the relationship between selfesteem, operationalized as an individual's negative or positive self-evaluation (Smith, Mackie, \& 
Claypool, 2014), suggest people scoring low on measures of self-esteem are prone to use more social media, often with the intention of enhancing self-image (Błachnio, Przepiorka, \& Rudnicka, 2016). It is necessary to point out that the association concerning compulsive use of social networking sites and selfworth is robust. For instance, a recent study of Polish Facebook addicts established that addicts had pointedly lower self-esteem than non-addicts (Błachnio et al., 2016) and, in a separate study, researchers found that people with low self-esteem use social networking sites to compensate, obtaining more popularity and friends in the virtual world (Mehdizadeh, 2010).

Social comparison and body image, especially in adolescents, tends to be closely tied to individual levels of self-worth. Social media apps such as Instagram and Tinder have negative implications in terms of appearance internalization and self-objectification (Lyu, 2016). A recent study on Tinder revealed that, regardless of gender, Tinder users reported significantly lower levels of satisfaction with their appearance and exhibited higher levels of body shaming, appearance internalization, and appearance comparison (Strubel \& Petrie, 2017). Additionally, studies indicate that Instagram plays a significant moderating role on self-esteem. Particularly, Instagram is influential when an individual's self-worth is contingent on external validation from social others (Stapleton, Luiz \& Chatwin, 2017). Further, Instagram, with its proliferation of attractive celebrity and peer images, amplifies women's body dissatisfaction through social comparison (Brown \& Tiggemann, 2016) and exposure to \#fitspiration on Instagram decreases body satisfaction in women (Prichard et al., 2017). These findings suggest that, although there is not always a direct relationship between media use and self-worth, social media addiction can be particularly detrimental to those already predisposed toward lower levels of self-esteem (Stapleton et al., 2017).

\section{Medium Differences}

Current studies suggest that the type of medium used moderates levels of self-esteem and its related factors, such as jealousy. Snapchat, a more popular app with younger audiences, elicits higher levels of jealousy than Facebook when users are exposed to potentially jealousy-provoking scenarios (Utz, Muscanell, \& Khalid, 2015). As a medium, Instagram encourages social comparison, a behavior that typically affects psychological well-being negatively. In a study analyzing the social media behavior of 18-29 year olds, researchers found that, generally, frequent Instagram use negatively affects those who follow more strangers in terms of social comparison and depressive tendencies (Lup, Trub, \& Rosenthal, 2015). In a similar respect, because Facebook users rarely post negative experiences, Facebook users are exposed to primarily positive relationships or the success of their Facebook friends. As such, employing Facebook for surveillance leads to Facebook envy, which, in turn, is related to depressive symptoms; this effect was not found for those who did not experience Facebook envy (Tandoc Jr., Ferrucci, \& Duffy, 2015).

Interestingly, social media addiction and gratifications vary across platforms. As an example, YouTube is found to be more addicting for content creators than for content viewers (Balakrishnan \& Griffiths, 2017). Additionally, gratification was essential for both content consumers and content purveyors in bolstering affinity toward YouTube; specifically, social gratification was found to be significantly related to higher levels of viewership as well as higher levels of creation (ibid). Scholars theorize that YouTube users could be using online relationships as a proxy for overcoming personal difficulties, such as introversion or aversion toward social expression (Ferris, 2001; Caplan \& High, 2010). If users are findings solace through emotional self-expression on YouTube, then this platform could potentially be less harmful to self-esteem compared to other social networking sites.

As a social medium, YouTube fundamentally differs from websites such as Instagram or Snapchat as YouTube supports, and encourages, long-form videos documenting anything from the video creators' thoughts to daily activities. Such videos, referred to in the community as "vlogs" (a combination of "video" and "blogs"), are equipped to facilitate deeper and more varied emotional expression than platforms such as Instagram. For instance, YouTube was found to function as a supportive environment to counter negative dominant narratives. Recent studies indicate that video logs created by Black adolescent girls on YouTube disseminated narratives of self-love and natural haircare, empowering young women to counter dominant aesthetic ideals (Phelps-Ward \& Laura, 2016). Perhaps the construction of YouTube, 
particularly YouTube video logs, lends itself to a supportive social environment for adolescent women. As such, YouTube videos, especially vlogs, may contain more wide-ranging emotional expression, with both negative and positive affective behaviors, which could lead to less self-destructive social comparison on the platform compared to other popular social networks.

Since much of the addictive behaviors related to social media use has to do with impression management and low-self-esteem, the authors hypothesize that YouTube content viewers with low selfesteem will have increased, or unchanging, levels of self-esteem post-exposure to YouTube vlogs compared to scrolling through Instagram or Facebook feeds. We theorize that YouTube viewers will be less susceptible to social comparison since on Instagram and Facebook, people tend to only post the highlights of their lives, sharing carefully curated positive moments, leading to the false perception that others' lives are more fulfilling than one's own. On YouTube, however, vloggers may appear more realistic due to the video format and they may be more willing to express negative emotions with their followers, which, in turn, could reduce the damage caused by social comparison since the video lends itself to reality, reducing the false impression of an ideal life fostered by other social platforms. Considering these assumptions, we hypothesize that:

$\boldsymbol{H}_{1}$ : Individuals will experience either an increase or no-change in self-esteem post exposure to YouTube vlogs

$\boldsymbol{H}_{2}:$ Individuals will experience a decrease in self-esteem post exposure to Instagram feeds

$\boldsymbol{H}_{3}$ : Individuals using YouTube will experience more positively affective emotions post-exposure to YouTube vlogs compared to individuals scrolling through Instagram feeds

\section{METHOD}

\section{Study 1}

Design

Study 1 will follow a 2 (platform: YouTube vs. Instagram) x 2(self-esteem: low vs. high) design to measure the interaction effect between platform use and self-esteem on the dependent measures of biased life-satisfaction and feelings of self-worth. The authors assume that individuals scoring low in levels of self-esteem will be more susceptible to social comparison compared to those with high self-esteem as past studies have demonstrated that these two variables correlate significantly with each other. Further, the authors examine how social propensity toward social comparison and external validation (high propensity vs. low propensity) may moderate the main effects of platform variation and self-esteem.

\section{Participants}

Roughly 150 adults will be recruited via a posting to participate for either $\$ 10$ or for extra credit in a Media class from a large Midwestern University. All participants will be required to supply answers to the Bergen social media addiction scale (BSMAS) as well as provide answers to measures of self-esteem (Rosenberg, 1965).

\section{Measures}

Bergen Social Media Addiction Scale

In line with previous research on social media addiction, the authors use the Bergen Social Media Addiction Scale (BSMAS), an adaptation of the validated Bergen Facebook Addiction Scale (Andreassen et al. 2012) to measure levels of addiction to social networking sites (Griffiths, 2005; Monacis et al., 2017). The BASMAS scale covers six items validated to reflect the central elements of general addiction, including salience, mood modification, conflict, withdrawal, and relapse (Griffiths, 2005). Respondents rated the frequency of occurrence on statements such as "How often during the last year have you become restless or troubled if you have been prohibited from using social media?" "How often in the last year 
have you used social media so much that it had a negative impact on your job/studies?" on a 5-point Likert scale from 1 (very rarely) to 5 (very often), with 3 (sometimes) as a neutral response (Andreassen et al., 2017; Monacis et al. 2017).

\section{Self-esteem}

Self-esteem was measured using the widely applied and validated Rosenberg Self-Esteem Scale (RSES), a Likert-type scale containing ten items on a four-point scale (Rosenberg, 1965). Replicating the procedure established by Rosenberg (1965), respondents rated statements such as "I feel that I have a number of good qualities" and "I certainly feel useless at times" on a scale ranging from 1 (strongly agree) to 4 (strongly disagree) with no natural 0 , or neutral point. Low scores on the RSES correlate with lower levels of self-esteem and high scores correlate with higher levels of self-esteem.

\section{Neuroticism}

Participants will be measured for neuroticism based on the big five personality measure that examines an individual's propensity toward introversion or extroversion, openness to new experience, neuroticism, agreeableness, and conscientiousness. The big five measures are commonly employed in psychological research and are often used as predictor variables to examine life stratification and subjective well-being. As such, this study utilizes the big five personality measures to examine an individual's level of neuroticism as an anticipated predictor variable for their subsequent levels of life-satisfaction.

\section{Materials}

The materials presented in study 1 will include content from popular Instagram pages and from popular YouTube vloggers. Respondents will be requested to browse media websites, like Instagram and YouTube, for roughly 15 minutes per platform. Respondents will either view Instagram content, a popular photo sharing social media app, or YouTube content, a popular video sharing platform. Additionally, some participants will be assigned to a control condition, where they will be required to browse an irrelevant website for the allotted time. Participants will be asked to browse the websites on a computer or phone provided by the researcher; this will ensure that the device, or technological medium itself, does not influence the participants in any way.

\section{Procedure}

Participants in study 1 will be recruited from a large, Midwestern university and will be payed roughly 10 USD or receive course credit, for their participation in the study. After signing the consent form, participants will complete demographic information (e.g., age, gender, education). The authors will also record participants' initial levels of self-esteem, neuroticism, life-satisfaction, and level of social media addiction prior to introducing them to the stimuli. Upon concluding the prerequisite questions, participants will be required to scroll through either Instagram or YouTube content for 15 minutes in each condition. Participants will be randomly assigned to either an Instagram, YouTube, or control condition. Those assigned to the Instagram condition will be required to view Instagram content for 15 minutes. Participants in the neutral condition will be asked to browse an irrelevant website that does not threaten self-esteem. Finally, those in the YouTube condition will view YouTube vlog content for 15 minutes.

\section{RESULTS}

The results of this study will suggest that the type of platform social media users engage with may either moderate, or even mediate, the influence of social media use on subsequent self-esteem and evaluations of life satisfaction. It is expected that participants exposed to the Instagram experimental condition will rate their life satisfaction lower in the post-measure than the pre-measure, and that they will also rate their levels of self-esteem lower in the post-measure compared to the pre-measure. Conversely, participants assigned to the YouTube condition will either rate their life satisfaction higher post-measure 
compared to pre-measure, or their will be no change between the pre-exposure and post-exposure to the stimulus.

Further, the relationship between life satisfaction, self-esteem, and social media platform will be articulated by how the medium is used for emotional self-expression. It is expected that because YouTube vloggers engage their audience through a video format, they are more likely to come across as "real" and they may express a broader range of emotions compared to Instagram users. Instagram, on the other hand, may have the opposite effect; that is, because people are only able to present themselves as an image or view only an image, the full range of a personality may be obstructed. Instagram users may be more sensitive to impression management and may express fewer emotions compared to YouTube vloggers.

\section{DISCUSSION}

The current study offers contributions to scholarship in media studies, communications, and psychology. This paper addresses a gap in the research as a robust body of literature exists on the influence of social media on psychological well-being, but few studies have examined the nuanced differences between social media platforms and their subsequent influence on psychological factors, such as self-esteem. This study explores how the medium itself, or the different type of social media platform used, could significantly influence established knowledge on the use of social media and its influence on life satisfaction and self-esteem. That is, this study aims to investigate the underlying factors of social media use that either bolster or attenuate psychological health.

Although several previous studies have explored either the empowering or the negative effects of social media use over time, few studies have explored the phenomenon from the perspective of medium differences; that is, the authors aim to delineate how the medium itself (i.e., the social media platform) may alter traditional perspectives on how social media influences self-esteem and life satisfaction. For instance, although social platforms such as Instagram may be related to lower self-esteem, platforms like YouTube may enhance it.

The findings of this study can be widely applied in modern business practice; the various social media are not monolithic. Because different platforms appear to impact and/or influence people in distinct ways, this variation needs to be recognized and addressed. This paper addresses a gap in the existing research stream on social media by examining the nuanced psychological effects of particular social media platforms with reference to self-esteem. In doing so, it begins a dialogue regarding how particular social media can either bolster or undercut psychological health.

Although earlier investigations have explored (1) the empowering influences of social media or (2) its negative effects, the implications of particular media differences has not been adequately addressed. By considering how particular media (i.e., the social media platform) exerts a specific influence, the traditional academic perspectives regarding the interconnections of social media, personality and attitude are usefully expanded. In the analysis provided above, for example, it appears that while Instagram may be related to lower self-esteem, YouTube tends to be connected to higher levels.

\section{CONCLUSION}

Strategic implications for both business and social policy are suggested by the distinctive psychological effects of specific social media platforms. When businesses promote their products and services, for example, considering the degree to which people have low or high self-esteem is an important variable. Some products, such as makeup and "beauty products" for example, are often sought by people (typically women) who are worried about their attractiveness (and, therefore, are concerned with self-esteem issues). By being aware that those who are involved with Instagram may experience self-esteem issues, companies that offer products that bolster self-esteem (by enhancing appearance) might benefit from promotional campaigns that are linked to Instagram.

Those involved with YouTube, in contrast, tend to experience higher degrees of self-esteem. As a result, products that cater to those with higher self-esteem may want to consider promotional campaigns 
that revolve around this social media platform. Athletic individuals who engage in competitive activities, such as sports, for example, often exhibit high self-esteem that is related to their willingness to accept challenges involving victory or loss. If companies that provide athletic equipment (such as running shoes) to such individuals develop a promotional campaign involving YouTube, therefore, a lucrative target markets might be more effectively addressed.

Those who are involved with human services (such as therapy and social work), furthermore, might benefit from knowing who uses particular social media. Psychological counseling, for example, often deals with self-esteem issues. If therapists gain a profile of the social media their clients utilize, a better understanding of their needs and vulnerabilities might result. Gaining access to a client's Instagram files, for example, might provide therapists with useful clues regarding self-esteem issues. This paper points to the potential analytic and diagnostic value of these tools.

This paper of course is explorative, not exhaustive and deals with only two social media platforms and how they are related to self-esteem. Nevertheless, this analysis demonstrates that the use of particular social media platforms is not a random affair. This reality has both theoretical and practitioner importance.

\section{Limitations and Future Research}

Although measures will be taken to ensure both the external and internal validity of this research, it faces certain limitations that must be accounted for in interpreting the data. Much of the analysis relies on participants' self-reports on their self-esteem, life satisfaction, social media consumption patterns, and social media addiction behaviors. Although validated scales for each of these variables will be used, respondents may not always be honest in a self-report. For instance, respondents may feel compelled to lie on a self-report on self-esteem or social media addiction as they may view certain tendencies as embarrassing.

Further, many people may not be particularly introspective, so it may be a difficult task for respondents to provide self-reports on their social media use or life satisfaction. In order to address these limitations, future research should combine self-reported measures of self-esteem with physiological measures. It would prove fruitful to examine how an individual's physiological responses will change depending on the social media platform they are engaging with; using physiological measures also addresses the limitations of self-reports since physiological responses are more immediate than selfreported responses.

\section{ACKNOWLEDGEMENT}

Tian Guang is the corresponding author: rgtian@yahoo.com. 


\section{REFERENCES}

Andreassen, C. S., \& Pallesen, S. (2014). Social network site addiction - An overview. Current Pharmaceutical Design, 20, 4053-4061.

Andreassen, C.S., Pallesen, S., \& Griffiths, M.D. (2017). The relationship between addictive use of social media, narcissism, and self-esteem: Findings from a large national survey. Addictive Behaviors, 64, $287-293$.

Andreassen, C.S., Torsheim, T., Brunborg, G.S., \& Pallesen. S. (2012). Development of a Facebook addiction scale. Psychological Reports, 110, 501-517.

Balakrishnan, J., \& Griffiths, M.D. (2017). Social media addiction: What is the role of content in YouTube? Journal of Behavioral Addictions, 6, 364-377.

Błachnio, A., Przepiorka, A., \& Rudnicka, P. (2016). Narcissism and self-esteem as predictors of dimensions of Facebook use. Personality and Individual Differences, 90, 296-301.

Blackwell, D., Leaman, C., Tramposch, R., Osborne, C., \& Liss, M. (2017). Extraversion, neuroticism, attachment style and fear of missing out as predictors of social media use and addiction. Personality and Individual Differences, 116, 69-72.

Brown, Z., \& Tiggemann, M. (2016). Attractive celebrity and peer images on Instagram: Effect on women's mood and body image. Body Image, 19, 37-43.

Caplan, S. E., \& High, A. C. (2010). Online social interaction, psychosocial well-being, and problematic Internet use. In K. Young \& C. Nabuco de Abreu (Eds.), Internet addiction: A handbook for evaluation and treatment (pp. 35-53). New York, NY: John Wiley \& Sons.

Ferris, J. (2001). Social ramifications of excessive Internet use among college-age males. Journal of Technology and Culture, 20, 44-53.

Griffiths, M. (2005). A “components" model of addiction within a biopsychosocial framework. Journal of Substance Abuse, 10, 191-197.

Griffiths, M. D., Kuss, D. J., \& Demetrovics, Z. (2014). Social networking addiction: An overview of preliminary findings. In K. P. Rosenberg, \& L. C. Feder (Eds.), Behavioral addictions: Criteria, evidence, and treatment (pp. 119-141). London, UK: Academic Press

Hawi, N.S., \& Samaha, M. (2017). The Relations Among Social Media Addiction, Self-Esteem, and Life Satisfaction in University Students. Social Science Computer Review, 35, 576-586.

Jenkins-Guarnieri, M. A., Wright, S. L., \& Hudiburgh, L. M. (2012). The relationships among attachment style, personality traits, interpersonal competency, and Facebook use. Journal of Applied Developmental Psychology, 33, 294-301.

Kuss, D. J., \& Griffiths, M. D. (2011). Online social networking and addiction: A literature review of empirical research. International Journal of Environmental and Public Health, 8, 3528-3552.

Kuss, D. J., Griffiths, M. D., Karila, L., \& Billieux, J. (2014). Internet addiction: A systematic review of epidemiological research for the last decade. Current Pharmaceutical Design, 20, 4026-4052.

Lin, J. H. (2015). The role of attachment style in Facebook use and social capital: Evidence from university students and a national sample. Cyberpsychology, Behavior, and Social Networking, $18,173-180$.

Lin, J. H. (2016). Need for relatedness: A self-determination approach to examining attachment styles, Facebook use, and psychological well-being. Asian Journal of Communication, 26, 153-173.

Lup, K., Trub, L., \& Rosenthal, L. (2015). Instagram \#Instasad?: Exploring Associations Among Instagram Use, Depressive Symptoms, Negative Social Comparison, and Strangers Followed. Cyberpsychology, Behavior, and Social Networking, 18, 247-252.

Lyu, O.L. (2017). Travel selfies on social media as objectified self-presentation. Tourism Management, $54,185-195$.

Mehdizadeh, S. (2010). Self-presentation 2.0: Narcissism and self-esteem on Facebook. CyberPsychology, Behavior, and Social Networking, 13, 357-364. 
Monacis, L., De Palo, V., Griffiths, M.D., \& Sintra, M. (2017). Social networking addiction, attachment style, and validation of the Italian version of the Bergan Social Media Addiction Scale. Journal of Behavior and Addictions, 6, 178-186.

Pantic, I. (2014). Online social networking and mental health. CyberPsychology, Behavior, and Social Networking, 17, 652-657.

Phelps-Ward, R., \& Laura, C. (2016). Talking back in cyberspace: self-love, hair care, and counter narratives in Black adolescent girls' YouTube vlogs. Gender and Education, 28, 807-820.

Prichard, I., McLachlan, A.C., Lavis, T., \& Tiggemann, M. (2017). The Impact of Different Forms of \#fitspiration Imagery on Body Image, Mood, and Self-Objectification among Young Women. Sex Roles, 1-11

Quan-Haase, A., \& Young, A. L. (2010). Uses and gratifications of social media: A comparison of Facebook and instant messaging. Bulletin of Science, Technology \& Society, 30, 350-361.

Quinn, K. (2016). Why We Share: A Uses and Gratifications Approach to Privacy Regulation in Social Media Use. Journal of Broadcasting \& Electronic Media, 60, 61-68.

Rosenberg, M. (1965). Society and the adolescent self-image. Princeton, NJ: Princeton University Press.

Stapleton, P., Luiz G., \& Chatwin H. (2017). Generation Validation: The Role of Social Comparison in Use of Instagram among Emerging Adults. Cyberpsychology, Behavior, and Social Networking, 20, 142-149.

Strubel, J., \& Petrie, T.A. (2017). Love me Tinder: Body image and psychosocial functioning among men and women. Body Image, 21, 34-38.

Tandoc Jr., Ferrucci, P., \& Duffy, M. (2015). Facebook use, envy, and depression among college students: Is facebooking depressing? Computers in Human Behavior, 43, 139-146.

Toma, C. L., \& Hancock, J. T. (2013). Self-affirmation underlies Facebook use. Personality \& Social Psychology Bulletin, 39, 321-331.

Utz, S., Muscanell, N., \& Khalid, C. (2015). Snapchat Elicits More Jealousy than Facebook: A Comparison of Snapchat and Facebook Use. CyberPsychology, Behavior, and Social Networking, $18,141-146$.

WeAreSocial. (2017). Social media use 2017. Retrieved December 2018, from https://wearesocial.com/special-reports/digital-in-2017-global-overview 